\title{
Acoustogalvanic Effect in Dirac and Weyl Semimetals
}

\author{
P. O. Sukhachov $\odot^{*}$ and H. Rostami $\odot^{\dagger}$ \\ Nordita, KTH Royal Institute of Technology and Stockholm University, Roslagstullsbacken 23, SE-106 91 Stockholm, Sweden
}

(Received 20 November 2019; accepted 3 March 2020; published 26 March 2020)

\begin{abstract}
The acoustogalvanic effect is proposed as a nonlinear mechanism to generate a direct electric current by passing acoustic waves in Dirac and Weyl semimetals. Unlike the standard acoustoelectric effect, which relies on the sound-induced deformation potential and the corresponding electric field, the acoustogalvanic one originates from the pseudoelectromagnetic fields, which are not subject to screening. The longitudinal acoustogalvanic current scales at least quadratically with the relaxation time, which is in contrast to the photogalvanic current where the scaling is linear. Because of the interplay of pseudoelectric and pseudomagnetic fields, the current could show a nontrivial dependence on the direction of sound wave propagation. Being within the experimental reach, the effect can be utilized to probe dynamical deformations and corresponding pseudoelectromagnetic fields, which are yet to be experimentally observed in Weyl and Dirac semimetals.
\end{abstract}

DOI: 10.1103/PhysRevLett.124.126602

Introduction.-The investigation of interplay between electric properties and sound waves has a long history and dates back to 1950s [1-5] (see also Refs. [6-8]). The generation of electric currents due to sound is known as the acoustoelectric effect. Its mechanism is related to a partial uncompensation of sound-induced dynamical deformation potential by electrons in solids. A sound wave drags charge carriers leading to a measurable current or voltage [3]. In low-dimensional systems, surface acoustic waves induced by piezoelectric substrate are routinely used to probe the acoustoelectric response [9-12]. Among them, a valley acoustoelectric effect driven by a surface acoustic wave was recently predicted in a two-dimensional (2D) semiconductor [13]. All of the previous proposals can be summarized as follows: sound waves induce an electric field interacting with charge carriers and resulting in an electric current. However, the possibility to generate currents via "fictitious" strain-induced electromagnetic fields that average to zero over the whole sample was not investigated before.

In recent years, there has been a surge of interest in the fictitious or pseudoelectromagnetic fields in one-, two-, and three-dimensional (3D) strained Dirac materials. As an example, we mention the pseudogauge field in carbon nanotubes [14], graphene [15-18], bilayer graphene $[19,20]$, and transition metal dichalcogenides (TMDs) $[21,22]$. A Hall current generated by a time-dependent pseudogauge field was also previously discussed in

Published by the American Physical Society under the terms of the Creative Commons Attribution 4.0 International license. Further distribution of this work must maintain attribution to the author(s) and the published article's title, journal citation, and DOI. Funded by Bibsam.

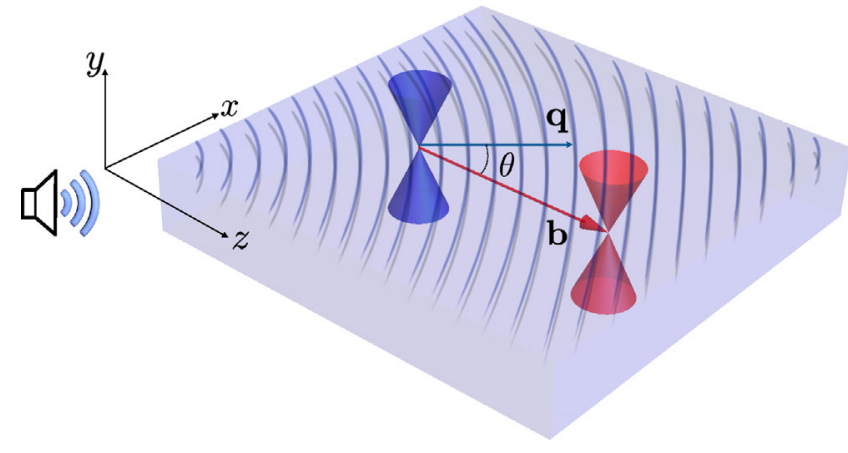

FIG. 1. The model setup of a WSM slab where the chiral shift $\mathbf{b}$ is directed along the $z$ axis and the wave vector of the sound wave $\mathbf{q}$ forms an angle $\theta$ with $\mathbf{b}$. Without the loss of generality, we assume that the vectors $\mathbf{q}$ and $\mathbf{b}$ are coplanar.

strained Dirac materials [23-25]. It is worth noting also that the pseudogauge fields can be generated in Weyl metamaterials [26-28]. In three dimensions, the possibility to produce axial gauge fields in strained Weyl semimetals (WSMs) can be noted [29-31]. Note that the appearance of strain-induced gauge fields is intimately connected with the fact that quasiparticles in Dirac and Weyl systems are described by the corresponding relativisticlike equations. Conventional metals with a parabolic band dispersion do not allow us to interpret strain in terms of gauge fields.

Dirac semimetals (DSMs) and WSMs represent a special class of solids with relativisticlike quasiparticles [32-35]. The valence and conduction bands touch at isolated Weyl nodes (Dirac points) allowing one to apply Weyl (Dirac) equations for the description of quasiparticle properties. If the time-reversal $(\mathcal{T})$ symmetry is broken, each Dirac point splits into two Weyl nodes of opposite chiralities 
separated by the vector $2 \mathbf{b}$ (known as the chiral shift [36]) in momentum space. As was shown in Refs. [29-31], mechanical strain in WSMs can lead to the generation of the axial gauge field $\mathbf{A}_{5}$. This field couples to the quasiparticles of opposite chirality as if they have opposite electric charges. The time-dependent and nonuniform $\mathbf{A}_{5}(\mathbf{r}, t)$ allows for the pseudoelectromagnetic fields $\left(c \mathbf{E}_{5}, \mathbf{B}_{5}\right)=\left(-\partial_{t} \mathbf{A}_{5}, \partial_{\mathbf{r}} \times \mathbf{A}_{5}\right)$. Certain DSM, such as $A_{3} \mathrm{Bi}(A=\mathrm{Na}, \mathrm{K}, \mathrm{Rb})$ and $\mathrm{Cd}_{3} \mathrm{As}_{2}[37,38]$ contain two overlapping copies of $\mathcal{T}$ symmetry broken WSMs with nonzero chiral shifts pointing in opposite directions and also allow for pseudo-electromagnetic fields. (In fact, these DSMs can be classified as $\mathbb{Z}_{2}$ WSMs [39].) Chiralityselective fields lead to many interesting phenomena [40]. Among them are the strain-induced chiral magnetic effect and the "negative" pseudomagnetic resistivity [41-45], quantum oscillations in pseudomagnetic fields [46], the chiral torsional effect [47-49], unusual collective excitations [50-52], axial analogs of the chiral separation and anomalous Hall effects [45], the lensing of Weyl quasiparticles [53-55], etc. However, to the best of our knowledge, the emergence of direct currents in WSMs and DSMs due to sound-induced dynamical strain fields was not discussed before.

The study of nonlinear processes such as the photogalvanic (or photovoltaic) effect [56-58], where a direct electric current $(\mathrm{dc})$ is generated due to the rectification of driving electromagnetic waves, has recently attracted significant experimental attention in topological materials [59-63]. Motivated by these studies, we propose to use dynamical deformations (e.g., sound or acoustic wave) instead of light to generate a dc current. In analogy to the photogalvanic, we dub this phenomenon the acoustogalvanic effect. Acoustic waves leads to dynamical local deformations in materials, which are modeled with a propagating displacement vector, $\mathbf{u}=\operatorname{Re}\left[\mathbf{u}_{0} e^{i(\mathbf{q} \cdot \mathbf{r}-\omega t)}\right]$, where the sound frequency is $\omega=v_{s} q, \mathbf{q}$ is the wave vector, $v_{s}$ stands for the sound velocity, and $\mathbf{u}_{0}$ is the amplitude of the displacement vector. Then, the acoustogalvanic (AG) current is defined as the nonlinear response to the dynamical strain fields

$$
j_{a}^{\mathrm{dc}}=\chi_{a b c}^{\mathrm{AG}} u_{b} u_{c}^{*}
$$

where $\chi_{a b c}^{\mathrm{AG}}$ is the acoustogalvanic susceptibility. As we already mentioned above, strains couple as effective oscillating pseudoelectromagnetic fields $\mathbf{E}_{5}$ and $\mathbf{B}_{5}$ in WSMs and DSMs. In terms of these fields, the AG current (1) can be rewritten as

$j_{a}^{\mathrm{dc}}=\sigma_{a b c} E_{5, b} E_{5, c}^{*}+\kappa_{a b c} \operatorname{Re}\left[E_{5, b} B_{5, c}^{*}\right]+\gamma_{a b c} B_{5, b} B_{5, c}^{*}$.

Note that due to the combined effect of the Berry curvature and pseudoelectromagnetic fields, there will be also alternating currents (ac) in the first order response (see Sec. S II.
A in the Supplemental Material [64]). In particular, they are related to the pseudomagnetic analog of the chiral magnetic effect $[42,45]$. However, due to their alternating nature and a different direction of these currents, they can be easily distinguished from the dc response and will not be considered here.

In what follows, we demonstrate that the pseudoelectromagnetic fields lead to a nontrivial AG response of WSMs and DSMs, where a dc current is generated in second order processes. By using the chiral kinetic theory $[50,71-74]$ as well as applying longitudinal sound waves, we calculate the intraband contribution to the AG current in a doped $\mathcal{T}$ symmetry-broken WSMs and certain DSMs [75]. As for possible material realizations of the present setup, we mention the WSM $\mathrm{EuCd}_{2} \mathrm{As}_{2}$ [76,77] and the DSMs $A_{3} \mathrm{Bi}(A=\mathrm{Na}, \mathrm{K}, \mathrm{Rb})$ and $\mathrm{Cd}_{3} \mathrm{As}_{2}[37,38]$. The origin of strain-induced electric currents is related to the acoustoelectric drag effect, where the AG current vanishes when the wave vector of sound wave goes to zero $q \rightarrow 0$. In addition, an interplay of the strain-induced pseudoelectric and pseudomagnetic fields allows for a nontrivial dependence of the AG current on the direction of sound wave propagation. Finally, we provide estimations of the proposed acoustogalvanic effect. As for the practical implications, we believe that it can be useful for investigating dynamical deformations. Finally, while we concentrate on the case of rectified electric current, the dc chiral current is also possible and is discussed in the Supplemental Material [64].

Model.-An effective Hamiltonian of strained WSMs in the vicinity of Weyl nodes is given by

$$
\mathcal{H}_{\lambda}=\lambda v_{F} \boldsymbol{\sigma} \cdot\left[\mathbf{p}+\frac{e}{c} \lambda \mathbf{A}_{5}(\mathbf{r}, t)\right]+D(\mathbf{r}, t),
$$

where $\lambda= \pm$ is chirality, $\sigma=\left(\sigma_{x}, \sigma_{y}, \sigma_{z}\right)$ is the vector of Pauli matrices, $\mathbf{p} \equiv-i \hbar \partial_{\mathbf{r}}$ is the momentum, $\mathbf{A}_{5}$ is the strain-induced gauge field, and $D$ is the deformation potential. It is easy to verify that the dispersion relation of the undeformed system in the vicinity of Weyl nodes is linear, $\epsilon_{\eta, \mathbf{p}}^{(0)}=\eta v_{F} p$, where $\eta=+$ and $\eta=-$ correspond to the conduction and valence bands, respectively.

For definiteness, we consider a longitudinal sound wave, i.e., $\mathbf{u}_{0}=u_{0} \hat{\mathbf{q}}$, and, without the loss of generality, set $\mathbf{b}=b \hat{\mathbf{z}}$. The corresponding model setup is presented in Fig. 1. The sound-induced deformation leads to the following axial gauge field $[31,41]$ :

$$
A_{5, i}=-\frac{c \hbar b}{e}\left[\beta u_{i z}+\tilde{\beta}(b) \delta_{i z} \sum_{j} u_{j j}\right] .
$$

The deformation potential $D$ can be represented as the series in displacement field $D=\sum_{n} D^{(n)}$. For instance, the deformation potential in the first order $n=1$ is 
$D^{(1)} \propto \sum_{j} u_{j j}$. Note that $u_{i j}=\left(\partial_{i} u_{j}+\partial_{j} u_{i}\right) / 2$ is the linearized strain tensor as well as $\beta$ and $\tilde{\beta}(b)$ are related to the Grüneisen parameters. Similarly to graphene [18], we assumed that the deformation potential $D$ is isotropic and momentum independent. The electric current $\mathbf{j}$ and charge $\rho$ densities are defined as $[71,73,78]$

$\mathbf{j}=-e \sum_{\lambda, \eta= \pm} \sum_{\mathbf{p}} \eta\left[\left(\partial_{t} \mathbf{r}\right) L_{\eta, \lambda} f_{\eta, \lambda}+\partial_{\mathbf{r}} \times\left(\epsilon_{\eta, \mathbf{p}} \boldsymbol{\Omega}_{\eta, \lambda} f_{\eta, \lambda}\right)\right]$,

$\rho=-e \sum_{\lambda, \eta= \pm} \sum_{\mathbf{p}} \eta L_{\eta, \lambda} f_{\eta, \lambda}$

with $\sum_{\mathbf{p}} \equiv \int d^{3} p /(2 \pi \hbar)^{3}$. Note that $L_{\eta, \lambda}=1-e \lambda\left(\mathbf{B}_{5} \cdot \boldsymbol{\Omega}_{\eta, \lambda}\right) / c$ stands for the phase-space volume, which is renormalized by the Berry curvature $\boldsymbol{\Omega}_{\eta, \lambda}=\sum_{n} \boldsymbol{\Omega}_{\eta, \lambda}^{(n)}$. In undeformed systems, the latter has a monopolelike structure $\boldsymbol{\Omega}_{\eta, \lambda}^{(0)}=$ $\lambda \eta \hat{\mathbf{p}} /\left(2 p^{2}\right)$ (see Sec. S I. in the Supplemental Material [64] for the fields-induced corrections to the Berry curvature). The last term in the electric current $\mathbf{j}$ corresponds to the orbital magnetization $\left(\propto \partial_{\mathbf{r}} \times \mathbf{M}\right)$ (see, e.g., Ref. [78]). The distribution function $f_{\eta, \lambda}$ for quasiparticles of each chirality is obtained by solving the Boltzmann equation in the presence of both pseudoelectric and pseudomagnetic fields,

$$
\partial_{t} f_{\eta, \lambda}+\left(\partial_{t} \mathbf{p}\right) \cdot \partial_{\mathbf{p}} f_{\eta, \lambda}+\left(\partial_{t} \mathbf{r}\right) \cdot \partial_{\mathbf{r}} f_{\eta, \lambda}=-\frac{f_{\eta, \lambda}-f_{\eta, \lambda}^{(0)}}{\tau} .
$$

Here $f_{\eta, \lambda}^{(0)}=1 /\left[e^{\eta\left(\epsilon_{\eta, p}+D-\mu_{\lambda}\right) / T}+1\right]$ is the local equilibrium distribution function where the divergent vacuum contribution was subtracted, $T$ is temperature in the energy units, and $\mu_{\lambda}$ is the chemical potential, which contains corrections from displacement fields, i.e., $\mu_{\lambda}=\sum_{n} \mu_{\lambda}^{(n)}$ where $\mu^{(0)}=\mu$. It can be decomposed into electric $\mu^{(n)}$ and chiral $\mu_{5}^{(n)}$ parts: $\mu_{\lambda}^{(n)}=\mu^{(n)}+\lambda \mu_{5}^{(n)}$. Note also that the quasiparticle dispersion obtains additional field-induced corrections $\epsilon_{\eta, \mathbf{p}}=\sum_{n} \epsilon_{\eta, \mathbf{p}}^{(n)}$ in the presence of pseudoelectromagnetic fields $[79,80]$ (for an explicit expression, see Sec. S I. in the Supplemental Material [64]). For simplicity, we utilized a simple relaxation time approximation for the collision integral, where $\tau$ is the intranode relaxation time and the internode processes were neglected. The equations of motion for the chiral quasiparticles are strongly modified by the Berry curvature and read as [78]

$$
\begin{aligned}
\partial_{t} \mathbf{r} & =\frac{\mathbf{v}_{\eta, \mathbf{p}}-e\left(\tilde{\mathbf{E}}_{\lambda} \times \boldsymbol{\Omega}_{\eta, \lambda}\right)-\lambda \frac{e}{c}\left(\mathbf{v}_{\eta, \mathbf{p}} \cdot \boldsymbol{\Omega}_{\eta, \lambda}\right) \mathbf{B}_{5}}{L_{\eta, \lambda}}, \\
\partial_{t} \mathbf{p} & =-\frac{e \tilde{\mathbf{E}}_{\lambda}+\lambda \frac{e}{c}\left(\mathbf{v}_{\eta, \mathbf{p}} \times \mathbf{B}_{5}\right)}{L_{\eta, \lambda}}
\end{aligned}
$$

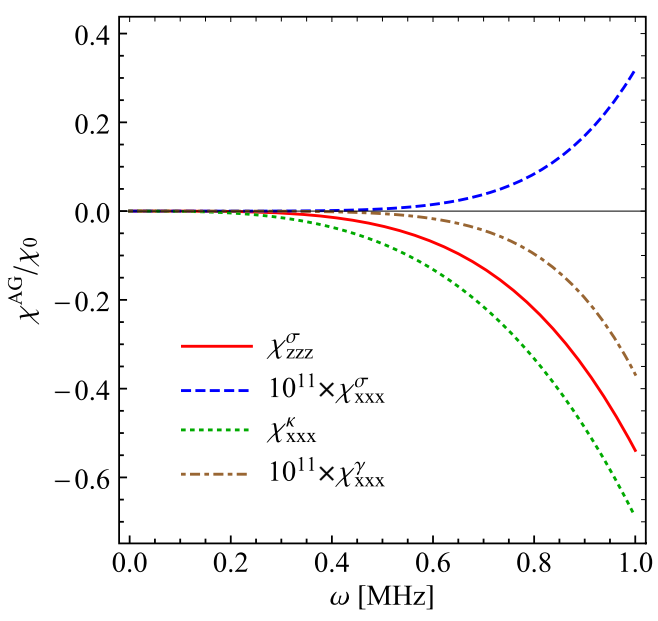

FIG. 2. The dependency of the acoustogalvanic susceptibility $\chi^{\mathrm{AG}}$ on the sound frequency $\omega$ for $\mathbf{q} \| \mathbf{b}(\theta=0)$ (solid red curve) and $\mathbf{q} \perp \mathbf{b}(\theta=\pi / 2)$ (the other three curves). Here $\chi_{0}=10^{4} \mathrm{~A} / \mathrm{cm}^{4}$.

where $\mathbf{v}_{\eta, \mathbf{p}}=\partial_{\mathbf{p}} \epsilon_{\eta, \mathbf{p}}$ stands for the quasiparticle velocity. Note also that the effective pseudoelectric field is $\tilde{\mathbf{E}}_{\lambda}=$ $\lambda \mathbf{E}_{5}+\partial_{\mathbf{r}}\left(D-\mu_{\lambda}\right) / e$ and we took into account that for a longitudinal sound wave $\tilde{\mathbf{E}}_{5} \cdot \mathbf{B}_{5}=0$.

Having defined the key aspects of the model, let us discuss how to calculate the current density. We assume that the deformations are sufficiently weak to allow for a perturbative solution to the Boltzmann equation (6). Since the propagation of sound distorts the ionic lattice, electrons tend to compensate local deviations from the charge neutrality and modify local electric chemical potential. As follows from the continuity relation for an electric current, the deviations of electric chemical potential $\mu^{(n)}$ compensate the deformation potential $D^{(n)}$. For the model at hand, the compensation is exact in the first order $n=1$. Then, the continuity relation for a chiral current allows for residual corrections to the chiral chemical potential that renormalize the effective electric field as $\tilde{\mathbf{E}}_{\lambda} \rightarrow \lambda \tilde{\mathbf{E}}_{5}$, where $\tilde{\mathbf{E}}_{5}=\mathbf{E}_{5}-\partial_{\mathbf{r}} \mu_{5}^{(1)} / e$ at $n=1$ (see also Sec. S II. A in the Supplemental Material [64]). The corresponding chiral chemical potential at $\omega \tau \ll 1$ reads as

$$
\mu_{5}^{(1)} \approx-\frac{e \tau v_{F}^{2}(1+i \omega \tau)}{3 \omega}\left(\mathbf{E}_{5} \cdot \mathbf{q}\right) e^{i(\mathbf{q} \cdot \mathbf{r}-\omega t)}+\text { c.c. }
$$

Acoustogalvanic response.-In the case of an arbitrary direction of sound wave propagation, both pseudoelectric $\mathbf{E}_{5}$ and pseudomagnetic $\mathbf{B}_{5}$ fields are generated. While the general expressions for the response tensors $\sigma_{a b c}, \kappa_{a b c}$, and $\gamma_{a b c}$ are given in Sec. S II. B in the Supplemental Material [64], here we focus on two limiting cases of sound wave propagation with respect to the chiral shift: (i) $\mathbf{q} \| \mathbf{b}$ and (ii) $\mathbf{q} \perp \mathbf{b}$. They correspond to $\theta=0$ and $\theta=\pi / 2$, respectively, in Fig. 1. 
Case (i): Let us start with the case of the second-order response at $\mathbf{q} \| \mathbf{b}$. As is easy to verify by using Eq. (4), the pseudomagnetic field $\mathbf{B}_{5}$ is absent in this case and the pseudoelectric one $\mathbf{E}_{5}$ is directed along $\mathbf{q}$. The only relevant element of the nonlinear conductivity is $\sigma_{z z z}$. In the leading order in small $\omega \tau$ and large $v_{F} / v_{s}$ it reads as [64]

$$
\sigma_{z z z} \approx-\frac{e^{3}}{\hbar^{2}} \frac{v_{F}}{v_{s}} \frac{\mu \tau^{2}}{18 \pi^{2} \hbar}+\mathcal{O}\left[(\omega \tau)^{2}\right] .
$$

Intriguingly, we find that, due to the contribution of the chiral chemical potential (9), the nonlinear conductivity here scales as $\tau^{2}$ and it is independent of $\omega$. Such a dependence on frequency is clearly different from that for the conventional optical rectification, where $\sigma^{\mathrm{PG}} \propto \tau$ or $1 / \omega$ [81-85]. The corresponding component of the acoustogalvanic response function $\chi_{z z z}^{\mathrm{AG}}$ follows [64]

$$
\chi_{z z z}^{\mathrm{AG}}=\frac{\omega^{4}}{v_{s}^{2}} \frac{\hbar^{2} b^{2}}{e^{2}}[\beta+\tilde{\beta}(b)]^{2} \sigma_{z z z} .
$$

As one can see, $\chi_{z z z}^{\mathrm{AG}}$ grows with the sound frequency as $\omega^{4}$ owing to quadratic dependence of the pseudoelectric field, $E_{5} \propto \omega^{2}$. Such a strong frequency dependence is one of the characteristic features of the acoustogalvanic response.

Case (ii): In the case $\mathbf{q} \perp \mathbf{b}$ (without the loss of generality, $\mathbf{q} \| \hat{\mathbf{x}}$ ), both $\mathbf{E}_{5}$ and $\mathbf{B}_{5}$ are nonzero, which enriches the dynamics of the system. In the leading order in $\omega \tau$, the following components of the response tensors are relevant [64]

$$
\begin{aligned}
& \sigma_{x z z} \approx \frac{e^{3}}{\hbar^{2}} \frac{v_{F}}{v_{s}} \frac{\mu \tau^{2}}{30 \pi^{2} \hbar}(\omega \tau)^{2}+\mathcal{O}\left[(\omega \tau)^{3}\right], \\
& \kappa_{x z y} \approx \frac{e^{3}}{\hbar^{2}} \frac{v_{F}}{c} \frac{\mu \tau^{2}}{12 \pi^{2} \hbar}+G_{1}\left(\mu, T, \Lambda_{\mathrm{IR}}\right)+\mathcal{O}\left[(\omega \tau)^{2}\right], \\
& \gamma_{x y y} \approx(\omega \tau)^{2} G_{2}(\mu, T)+\mathcal{O}\left[(\omega \tau)^{3}\right] .
\end{aligned}
$$

The explicit definitions of $G_{1}\left(\mu, T, \Lambda_{\mathrm{IR}}\right)$ and $G_{2}(\mu, T)$ are given in Eqs. (S71) and (S72) in the Supplemental Material [64]. Note that, in the second-order chiral kinetic theory, function $G_{1}\left(\mu, T, \Lambda_{\mathrm{IR}}\right)$ depends on the regularization scheme. While we used a simple infrared cutoff $\Lambda_{\mathrm{IR}}$, a more refined treatment might be required (see also the discussion in Sec. S II. B in the Supplemental Material [64]). As one can see, the leading order contribution in $\omega \tau \ll 1$ stems from the interplay between the pseudoelectric and pseudomagnetic fields quantified by $\kappa_{x z y}$ in Eq. (13). Unlike the response to the pseudoelectric fields, where, as in the case (i), electric current is also directed along the chiral shift, $\kappa_{x z y}$ is related to the Hall-like response $\propto \mathbf{E}_{5} \times \mathbf{B}_{5}$. Note also that this part of the response contains terms insensitive to the relaxation time.

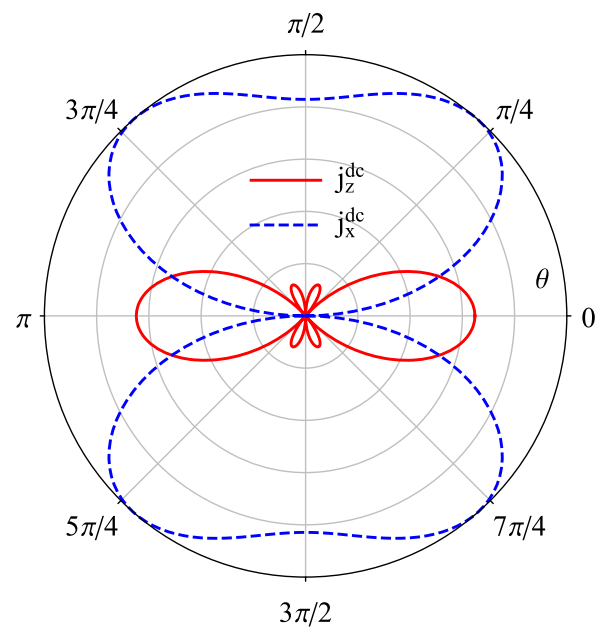

FIG. 3. The dependency of the rectified current components $j_{z}^{\mathrm{dc}}$ and $j_{x}^{\mathrm{dc}}$ on the angle $\theta$ between the chiral shift $\mathbf{b}$ and the wave vector q. We fixed $\omega=1 \mathrm{MHz}$. A typical value of the rectified current is $j^{\mathrm{dc}} \approx 54 u_{0}^{2} / \mu \mathrm{m}^{2}\left[\mu \mathrm{A} / \mathrm{cm}^{2}\right]$ at $\theta=0$.

The corresponding acoustogalvanic susceptibility contains three contributions $\chi_{x x x}^{\mathrm{AG}}=\chi_{x x x}^{\sigma}+\chi_{x x x}^{\kappa}+\chi_{x x x}^{\gamma}$. Explicit expressions for these terms are given in Eqs. (S68)-(S70) in the Supplemental Material [64]. Their numerical values are depicted in Fig. 2. The difference in magnitude between $\chi_{x x x}^{\sigma}$ and $\chi_{x x x}^{\gamma}$ as well as $\chi_{x x x}^{\kappa}$ is related to the fact that the relative scaling of the former with respect to the latter is $(\omega \tau)^{2}$ for small $\omega \tau \ll 1$. In addition, the strong frequency dependence of the acoustogalvanic susceptibility is clearly evident from the figure. Furthermore, we present the dependence of the current components $j_{x}^{\mathrm{dc}}$ and $j_{z}^{\mathrm{dc}}$ on the angle between the sound wave vector and the chiral shift in Fig. 3. While the angular profile of the former component is $\sim \sin \theta\left[1+A_{0} \cos (2 \theta)\right], j_{z}^{\mathrm{dc}} \propto \cos \theta\left[1-A_{0} \cos (2 \theta)\right]$, where $A_{0}$ is a combination of functions $G_{1}\left(\mu, T, \Lambda_{\mathrm{IR}}\right), G_{2}(\mu, T)$, and terms $\propto \mu$. The terms with $\cos (2 \theta)$ cause a nontrivial modulation observed in Fig. 3. In particular, due to the interplay of the pseudoelectric and pseudomagnetic fields, $j_{x}^{\text {dc }}$ attains its maximal values at $\theta \approx \pi / 4$ and has a characteristic butterflylike angular profile. For our numerical estimates, we used the numerical parameters valid for the DSM $\mathrm{Cd}_{3} \mathrm{As}_{2}$ [86-90]: $v_{F} \approx 1.5 \times 10^{8} \mathrm{~cm} / \mathrm{s}, \mu \approx$ $200 \mathrm{meV}, b \approx 1.6 \mathrm{~nm}^{-1}, v_{s} \approx 2.3 \times 10^{5} \mathrm{~cm} / \mathrm{s}$, and $\tau \approx 1 \mathrm{ps}$. In addition, we assume $\beta \approx 1, T=5 \mathrm{~K}$, and $\tilde{\beta}(b) \approx 1$.

Discussion and summary.-In this study, a nonlinear mechanism to generate a rectified electric current by passing sound waves in WSMs and certain DSMs is proposed. Unlike the conventional acoustoelectric effect, the dc current is produced by the strain-induced pseudoelectromagnetic fields rather than real electric fields. Therefore, in analogy to the photogalvanic effect, we called this mechanism the acoustogalvanic rectification.

The sound-induced dc current quickly grows with a sound frequency. This profound difference to the usual 
optical rectification is explained by the fact that the pseudoelectromagnetic fields are determined by the dynamics of the deformation vector and, therefore, grow with frequency. By using the realistic model parameters, we estimated that the acoustogalvanic current should be experimentally observable for high frequencies (e.g., ultrasound) and amplitudes of sound. Indeed, the order of magnitude of the AG current is $I^{\mathrm{dc}} \sim 100 \mathrm{nA}$ for $\omega=10 \mathrm{MHz}, u_{0}=10 \mathrm{~nm}$, and mm-sized crystals. For example, photocurrents of such magnitudes were recently observed in, e.g., Ref. [63]. Such a current is also comparable to that in TMDs, where $I^{\mathrm{dc}} \sim 10 \mu \mathrm{A}$ for $\mathrm{GHz}$ frequencies [13], and in graphene, where $I^{\mathrm{dc}} \sim$ $0.1-1 \mu \mathrm{A}$ for $\mathrm{MHz}$ frequencies [12]. As an additional advantage over the acoustoelectric effect, which relies on an electric field, pseudoelectromagnetic fields that drive acoustogalvanic currents are not subject to screening and could attain significantly high values.

Let us briefly comment on the case of DSMs such as $\mathrm{Na}_{3} \mathrm{Bi}$ and $\mathrm{Cd}_{3} \mathrm{As}_{2}$ where both $\mathcal{T}$ and parity $(\mathcal{P})$ symmetries are preserved. In each copy of WSMs that constitute these DSMs, the direction of pseudoelectromagnetic fields is opposite and cancels the majority of first-order effects such as, e.g., the anomalous Hall effect. However, the second-order response will be doubled with respect to simple $\mathcal{T}$ symmetry broken WSMs.

Further, we discuss the AG effect when $\mathcal{T}$ symmetry is preserved but $\mathcal{P}$ symmetry is broken. By using a simple model where the nondegenerate Weyl nodes are separated only in energy by $2 b_{0}$, it can be shown that there will be the scalar component of the gauge field $A_{0.5}$ [64]. The direction of the corresponding pseudoelectric field $\mathbf{E}_{5}=-\partial_{\mathbf{r}} A_{0,5}$ is determined exclusively by the sound wave vector. Then, the susceptibility tensor is isotropic $\chi_{a b c}^{\mathrm{AG}} \sim$ $-\delta_{a b} \delta_{b c} b_{0}^{2} v_{F} \mu \tau^{2} \omega^{4} / v_{s}^{5}$ and can be comparable in magnitude to that in Fig. 2. In the case of WSMs with multiple pairs of Weyl nodes, such as transition metal monopnictides (e.g., TaAs), AG currents should be generated independently for each pair. Therefore, the rectified current $\mathbf{j}^{\mathrm{dc}}$ should be present regardless of the direction of the wave vector q. In addition, the acoustogalvanic effect should occur in type-II WSMs [91], as long as strains can be interpreted in terms of axial gauge fields [64]. Thus, the existence of the acoustogalvanic effect relies primarily on whether strains can be interpreted as axial gauge fields rather than specific properties of materials.

Finally, while in this study we concentrated on the case of 3D WSMs, we believe that our qualitative results can be applied for 2D Dirac materials such as graphene and TMDs. Indeed, since dynamical strain also generates pseudoelectric fields in these materials, one can follow the same steps in the calculation of acoustogalvanic response as discussed in our study. Thus, a dc current could be also generated in 2D materials due to the acoustic drag effect.
We are grateful to A. V. Balatsky for useful discussions. P. O. S. thanks E. V. Gorbar for critical comments. We acknowledge the support from the VILLUM FONDEN via the Centre of Excellence for Dirac Materials (Grant No. 11744), the European Research Council under the European Unions Seventh Framework ERS-2018-SYG 810451 HERO, and the Knut and Alice Wallenberg Foundation KAW 2018.0104. H. R. acknowledges support from the Swedish Research Council (VR 2018-04252).

*pavlo.sukhachov@su.se

†habib.rostami@su.se

[1] R. H. Parmenter, Phys. Rev. 89, 990 (1953).

[2] A. I. Akhiezer, M. I. Kaganov, and G. Ia. Liubarskii, J. Exp. Theor. Phys. 5, 685 (1957), http://www.jetp.ac.ru/cgi-bin/e/ index/e/5/4/p685?a=list.

[3] G. Weinreich and H. G. White, Phys. Rev. 106, 1104 (1957).

[4] V. G. Skobov and E. A. Kaner, J. Exp. Theor. Phys. 19, 189 (1964), http://www.jetp.ac.ru/cgi-bin/e/index/e/19/1/p189? $\mathrm{a}=$ list.

[5] S. G. Eckstein, J. Appl. Phys. 35, 2702 (1964).

[6] A. A. Abrikosov, Fundamentals of the Theory of Metals (North-Holland, Amsterdam, 1988).

[7] C. Kittel, Quantum Theory of Solids (Wiley, New York, 1987).

[8] V. V. Gudkov and J. D. Gavenda, Magnetoacoustic Polarization Phenomena in Solids (Springer Science and Business Media, 2000).

[9] A. Wixforth, J. Scriba, M. Wassermeier, J. P. Kotthaus, G. Weimann, and W. Schlapp, Phys. Rev. B 40, 7874 (1989).

[10] R. L. Willett, M. A. Paalanen, R. R. Ruel, K. W. West, L. N. Pfeiffer, and D. J. Bishop, Phys. Rev. Lett. 65, 112 (1990).

[11] V. I. Falko, S. V. Meshkov, and S. V. Iordanskii, Phys. Rev. B 47, 9910 (1993).

[12] A. Hernández-Mínguez, Y.-T. Liou, and P. V. Santos, J. Phys. D 51, 383001 (2018).

[13] A. V. Kalameitsev, V. M. Kovalev, and I. G. Savenko, Phys. Rev. Lett. 122, 256801 (2019).

[14] C. L. Kane and E. J. Mele, Phys. Rev. Lett. 78, 1932 (1997).

[15] H. Suzuura and T. Ando, Phys. Rev. B 65, 235412 (2002).

[16] K. Sasaki, Y. Kawazoe, and R. Saito, Prog. Theor. Phys. 113, 463 (2005).

[17] M. I. Katsnelson and K. S. Novoselov, Solid State Commun. 143, 3 (2007).

[18] M. A. H. Vozmediano, M. I. Katsnelson, and F. Guinea, Phys. Rep. 496, 109 (2010).

[19] E. Mariani, A. J. Pearce, and F. von Oppen, Phys. Rev. B 86, 165448 (2012).

[20] H. Rostami and R. Asgari, Phys. Rev. B 88, 035404 (2013).

[21] M. A. Cazalilla, H. Ochoa, and F. Guinea, Phys. Rev. Lett. 113, 077201 (2014).

[22] H. Rostami, R. Roldàn, E. Cappelluti, R. Asgari, and F. Guinea, Phys. Rev. B 92, 195402 (2015).

[23] A. Vaezi, N. Abedpour, R. Asgari, A. Cortijo, and M. A. H. Vozmediano, Phys. Rev. B 88, 125406 (2013).

[24] F. von Oppen, F. Guinea, and E. Mariani, Phys. Rev. B 80, 075420 (2009). 
[25] E. Sela, Y. Bloch, F. von Oppen, and M. B. Shalom, Phys. Rev. Lett. 124, 026602 (2020).

[26] S. Roy, M. Kolodrubetz, N. Goldman, and A. G. Grushin, 2D Mater. 5, 024001 (2018).

[27] V. Peri, M. Serra-Garcia, R. Ilan, and S. D. Huber, Nat. Phys. 15, 357 (2019).

[28] H. Jia, R. Zhang, W. Gao, Q. Guo, B. Yang, J. Hu, Y. Bi, Y. Xiang, C. Liu, and S. Zhang, Science 363, 148 (2019).

[29] Z. Jian-Hui, J. Hua, N. Qian, and S. Jun-Ren, Chin. Phys. Lett. 30, 027101 (2013).

[30] M. A. Zubkov, Ann. Phys. (N.Y.) 360, 655 (2015).

[31] A. Cortijo, Y. Ferreirós, K. Landsteiner, and M. A. H. Vozmediano, Phys. Rev. Lett. 115, 177202 (2015).

[32] T. O. Wehling, A. M. Black-Schaffer, and A. V. Balatsky, Adv. Phys. 63, 1 (2014).

[33] B. Yan and C. Felser, Annu. Rev. Condens. Matter Phys. 8, 337 (2017).

[34] M. Z. Hasan, S.-Y. Xu, I. Belopolski, and C.-M. Huang, Annu. Rev. Condens. Mattter Phys. 8, 289 (2017).

[35] N. P. Armitage, E. J. Mele, and A. Vishwanath, Rev. Mod. Phys. 90, 015001 (2018).

[36] E. V. Gorbar, V. A. Miransky, and I. A. Shovkovy, Phys. Rev. C 80, 032801(R) (2009).

[37] Z. Wang, Y. Sun, X.-Q. Chen, C. Franchini, G. Xu, H. Weng, X. Dai, and Z. Fang, Phys. Rev. B 85, 195320 (2012).

[38] Z. Wang, H. Weng, Q. Wu, X. Dai, and Z. Fang, Phys. Rev. B 88, 125427 (2013).

[39] E. V. Gorbar, V. A. Miransky, I. A. Shovkovy, and P. O. Sukhachov, Phys. Rev. B 91, 121101(R) (2015).

[40] R. Ilan, A. G. Grushin, and D. I. Pikulin, Nat. Rev. Phys. 2, 29 (2020).

[41] A. Cortijo, D. Kharzeev, K. Landsteiner, and M. A. H. Vozmediano, Phys. Rev. B 94, 241405(R) (2016).

[42] A. G. Grushin, J. W. F. Venderbos, A. Vishwanath, and R. Ilan, Phys. Rev. X 6, 041046 (2016).

[43] D. I. Pikulin, A. Chen, and M. Franz, Phys. Rev. X 6, 041021 (2016).

[44] M. N. Chernodub and M. A. Zubkov, Phys. Rev. B 95, 115410 (2017).

[45] Z.-M. Huang, J. Zhou, and S.-Q. Shen, Phys. Rev. B 96, 085201 (2017).

[46] T. Liu, D. I. Pikulin, and M. Franz, Phys. Rev. B 95, 041201 (R) (2017).

[47] O. Parrikar, T. L. Hughes, and R. G. Leigh, Phys. Rev. D 90 , 105004 (2014).

[48] H. Sumiyoshi and S. Fujimoto, Phys. Rev. Lett. 116, 166601 (2016).

[49] Z. V. Khaidukov and M. A. Zubkov, JETP Lett. 108, 670 (2018).

[50] E. V. Gorbar, V. A. Miransky, I. A. Shovkovy, and P. O. Sukhachov, Phys. Rev. Lett. 118, 127601 (2017).

[51] E. V. Gorbar, V. A. Miransky, I. A. Shovkovy, and P. O. Sukhachov, Phys. Rev. B 95, 115202 (2017); 95, 115422 (2017); 95, 205141 (2017).

[52] M. N. Chernodub and M. A. H. Vozmediano, Phys. Rev. Research 1, 032040 (2019).

[53] E. V. Gorbar, V. A. Miransky, I. A. Shovkovy, and P. O. Sukhachov, Phys. Rev. B 95, 241114(R) (2017).

[54] A. Westström and T. Ojanen, Phys. Rev. X 7, 041026 (2017).
[55] R. Soto-Garrido and E. Muñoz, J. Phys. Condens. Matter 30, 195302 (2018).

[56] V. I. Belinicher, Phys. Lett. A 66, 213 (1978).

[57] E. L. Ivchenko and G. E. Pikus, JETP Lett. 27, 604 (1978), http://www.jetpletters.ac.ru/ps/1554/article_23792.shtml.

[58] V. I. Belinicher and B. I. Belinicher, Sov. Phys. Usp. 23, 199 (1980).

[59] Q. Ma, S.-Y. Xu, C.-K. Chan, C.-L. Zhang, G. Chang, Y. Lin, W. Xie, T. Palacios, H. Lin, S. Jia, P. A. Lee, P. Jarillo-Herrero, and N. Gedik, Nat. Phys. 13, 842 (2017).

[60] G. B. Osterhoudt, L. K. Diebel, M. J. Gray, X. Yang, J. Stanco, X. Huang, B. Shen, N. Ni, P. J. W. Moll, Y. Ran, and K. S. Burch, Nat. Mater. 18, 471 (2019).

[61] N. Sirica, R. I. Tobey, L. X. Zhao, G. F. Chen, B. Xu, R. Yang, B. Shen, D. A. Yarotski, P. Bowlan, S. A. Trugman, J.-X. Zhu, Y. M. Dai, A. K. Azad, N. Ni, X. G. Qiu, A. J. Taylor, and R. P. Prasankumar, Phys. Rev. Lett. 122, 197401 (2019).

[62] Z. Ji, G. Liu, Z. Addison, W. Liu, P. Yu, H. Gao, Z. Liu, A. M. Rappe, C. L. Kane, E. J. Mele, and R. Agarwal, Nat. Mater. 18, 955 (2019).

[63] J. Ma, Q. Gu, Y. Liu, J. Lai, P. Yu, X. Zhuo, Z. Liu, J.-H. Chen, J. Feng, and D. Sun, Nat. Mater. 18, 476 (2019).

[64] See Supplemental Material at http://link.aps.org/ supplemental/10.1103/PhysRevLett.124.126602 for details of the chiral kinetic theory, calculation of the second-order electric and chiral response, and the spectrum of a type-II model, which includes Refs. [65-70].

[65] K. Landsteiner, Acta Phys. Polon. B 47, 2617 (2016).

[66] A. Polkovnikov, Ann. Phys. (N.Y.) 325, 1790 (2010).

[67] M. M. Vazifeh and M. Franz, Phys. Rev. Lett. 111, 027201 (2013).

[68] H. Shapourian, T. L. Hughes, and S. Ryu, Phys. Rev. B 92, 165131 (2015).

[69] A. Cortijo and M. A. Zubkov, Ann. Phys. (N.Y.) 366, 45 (2016).

[70] M. A. Zubkov and M. Lewkowicz, Ann. Phys. (N.Y.) 399, 26 (2018).

[71] D. T. Son and N. Yamamoto, Phys. Rev. D 87, 085016 (2013).

[72] M. A. Stephanov and Y. Yin, Phys. Rev. Lett. 109, 162001 (2012).

[73] D. T. Son and N. Yamamoto, Phys. Rev. Lett. 109, 181602 (2012).

[74] D. T. Son and B.Z. Spivak, Phys. Rev. B 88, 104412 (2013).

[75] The acoustogalvanic effect should be also present in other types of WSMs such as parity-symmetry broken and type-II ones as long as strains can be interpreted in terms of axial gauge fields.

[76] L.-L. Wang, N. H. Jo, B. Kuthanazhi, Y. Wu, R. J. McQueeney, A. Kaminski, and P. C. Canfield, Phys. Rev. B 99, 245147 (2019).

[77] J.-Z. Ma et al., Sci. Adv. 5, eaaw4718 (2019).

[78] D. Xiao, M.-C. Chang, and Q. Niu, Rev. Mod. Phys. 82, 1959 (2010).

[79] Y. Gao, S. A. Yang, and Q. Niu, Phys. Rev. Lett. 112, 166601 (2014). 
[80] Y. Gao, S. A. Yang, and Q. Niu, Phys. Rev. B 91, 214405 (2015).

[81] J. E. Sipe and A. I. Shkrebtii, Phys. Rev. B 61, 5337 (2000).

[82] F. de Juan, A. G. Grushin, T. Morimoto, and J. E. Moore, Nat. Commun. 8, 15995 (2017).

[83] H. Rostami and M. Polini, Phys. Rev. B 97, 195151 (2018).

[84] O. Matsyshyn and I. Sodemann, Phys. Rev. Lett. 123, 246602 (2019).

[85] F. de Juan, Y. Zhang, T. Morimoto, Y. Sun, J. E. Moore, and A. G. Grushin, Phys. Rev. Research 2, 012017 (2020).

[86] W. Freyland, C. Madelung, A. Goltzene, P. Grosse et al., Non-Tetrahedrally Bonded Elements and Binary Compounds I, Condensed Matter (Springer-Verlag Berlin Heidelberg, 1998).
[87] H. Wang, Y. Xu, M. Shimono, Y. Tanaka, and M. Yamazaki, Mater. Trans., JIM 48, 2349 (2007).

[88] M. Neupane, S.-Y. Xu, R. Sankar, N. Alidoust, G. Bian, C. Liu, I. Belopolski, T.-R. Chang, H.-T. Jeng, H. Lin, A. Bansil, F. Chou, and M.Z. Hasan, Nat. Commun. 5, 3786 (2014).

[89] Z. K. Liu, J. Jiang, B. Zhou, Z. J. Wang, Y. Zhang, H. M. Weng, D. Prabhakaran, S-K. Mo, H. Peng, P. Dudin, T. Kim, M. Hoesch, Z. Fang, X. Dai, Z. X. Shen, D. L. Feng, Z. Hussain, and Y. L. Chen, Nat. Mater. 13, 677 (2014).

[90] C.-Z. Li, L.-X. Wang, H. Liu, J. Wang, Z.-M. Liao, and D.-P. Yu, Nat. Commun. 6, 10137 (2015).

[91] A. A. Soluyanov, D. Gresch, Z. Wang, Q. Wu, M. Troyer, X. Dai, and B.A. Bernevig, Nature (London) 527, 495 (2015). 